\title{
Guidelines of the European Respiratory Society and the European Society of Thoracic Surgeons for the management of malignant pleural mesothelioma
}

\author{
A. Scherpereel, P. Astoul, P. Baas, T. Berghmans, H. Clayson, P. de Vuyst, \\ H. Dienemann, F. Galateau-Salle, C. Hennequin, G. Hillerdal, C. Le Péchoux, L. Mutti, \\ J-C. Pairon, R. Stahel, P. van Houtte, J. van Meerbeeck, D. Waller and W. Weder
}

ABSTRACT: Malignant pleural mesothelioma (MPM) is a rare tumour but with increasing incidence and a poor prognosis. In 2008, the European Respiratory Society/European Society of Thoracic Surgeons Task Force brought together experts to propose practical and up-to-dated guidelines on the management of MPM.

To obtain an earlier and reliable diagnosis of MPM, the experts recommend performing thoracoscopy, except in cases of pre-operative contraindication or pleural symphysis. The standard staining procedures are insufficient in $\sim 10 \%$ of cases. Therefore, we propose using specific immunohistochemistry markers on pleural biopsies. In the absence of a uniform, robust and validated staging system, we advice use of the most recent TNM based classification, and propose a three step pre-treatment assessment. Patient's performance status and histological subtype are currently the only prognostic factors of clinical importance in the management of MPM. Other potential parameters should be recorded at baseline and reported in clinical trials. MPM exhibits a high resistance to chemotherapy and only a few patients are candidates for radical surgery. New therapies and strategies have been reviewed.

Because of limited data on the best combination treatment, we emphasise that patients who are considered candidates for a multimodal approach should be included in a prospective trial at a specialised centre.

KEYWORDS: Asbestos, cancer, guidelines, mesothelioma, pleura, treatment

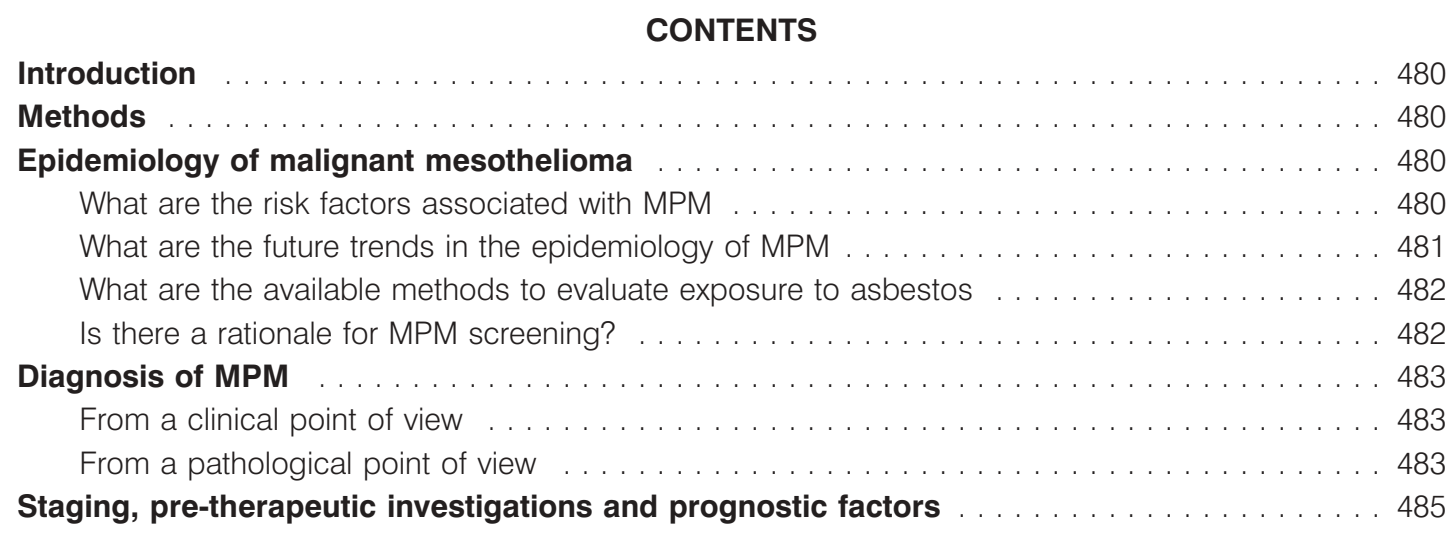

This article has supplementary data accessible from www.erj.ersjournals.com
AFFILIATIONS

For a full list of affiliations, please see the Acknowledgements section.

CORRESPONDENCE

A. Scherpereel Dept of Pulmonary and Thoracic Oncology, Hôpital Calmette CHRU of Lille 59037 Lille Cedex France

E-mail: a-scherpereel@chru-lille.fr

Received:

April 152009

Accepted after revision:

Aug 212009

First published online: Aug 282009 
Which staging classification is used? . . . . . . . . . . . 485

What are the minimal pre-treatment staging examinations? . . . . . . . . . . . . . . . . . . 485

Which prognostic factors are of importance? . . . . . 485

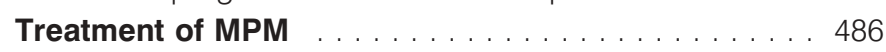

Surgery for MPM ................ 486

\section{INTRODUCTION}

Previously considered to be rare, malignant pleural mesothelioma (MPM) is a highly aggressive tumour that has become a very important issue over recent years [1]. Asbestos exposure is the main factor involved in pathogenesis, which can explain the rise in incidence of MPM since the 1960s. Despite the prohibition of asbestos use in Europe in 2005, as in most other developed countries, epidemiological projections estimate that the incidence of MPM is still increasing and will peak within the next 10 yrs [1, 2]. In addition, some countries still produce large amounts of asbestos, with the "top five" including Russia (which is by far the larger producer), China, Kazakhstan, Brazil and Canada. Asbestos is used in these countries as well as in other emerging countries, such as India and less-developed nations [3].

The diagnosis of MPM is difficult because the disease may occur up to 30-40 yrs after asbestos exposure, and the differential diagnosis on pleural biopsy between MPM and pleural benign disease or metastasis of adenocarcinoma may be difficult in some cases, even with the use of immunohistochemistry [4,5]. Since MPM patients have a poor outcome and an optimal treatment is not clearly defined, even in recent guidelines from the French speaking Society for Chest Medicine (SPLF), the British Thoracic Society (BTS) and the European Society of Medical Oncology (ESMO) [4-7], MPM will remain a major public-health problem for many years.

Therefore, the European Respiratory Society (ERS), in collaboration with the European Society of Thoracic Surgeons (ESTS), brought together experts on mesothelioma from different scientific societies between May 2007 and November 2008 to draw up recommendations in order to provide clinicians with clear, concise, up-to-date guidelines on the management of MPM.

\section{METHODS}

A systematic analysis of the literature from 1990 to 2009 was performed by the experts using the following databases: Medline (National Library of Medicine, USA), Embase (Elsevier, the Netherlands), Cochrane Library (UK), National Guideline Clearinghouse (USA), HTA Database (International Network of Agencies for Health Technology Assessment), National Institute of Health database (USA), International Pleural Mesothelioma Program (World Health Organization (WHO) Database). The following keywords were used as the search terms in the literature: pleura; cancer; mesothelioma; guidelines; asbestos; treatment; surgery; chemotherapy; and radiotherapy. However, the literature search for chemotherapy took place during the period from 1965 to 2009.

Each recommendation was graded by the experts based on the official proposal for evidence-based medicine, provided by the American College of Chest Physicians [8] (table 1 in the

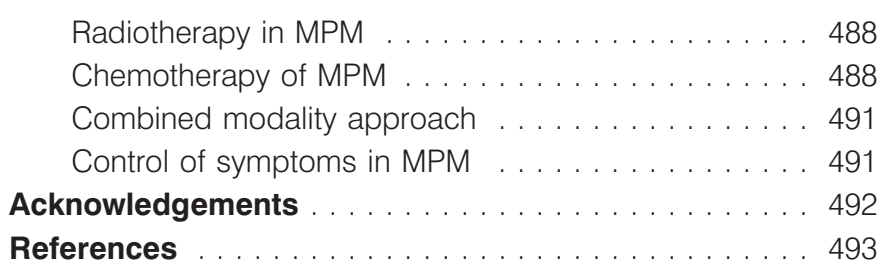

supplementary data). Briefly, the strength of any recommendation of the ACCP depends on the following factors: 1) the trade off between the benefits, the risks and the burdens (clear in category 1 , or not clear in category 2); and 2) the quality of the evidence regarding treatment effect, graded as follows: randomised controlled trials (RCT) that show consistent results, or observational studies with very strong treatment effects; RCT with limitations, or observational studies with exceptional strengths; and observational studies without exceptional strengths and case series. Thus, the ACCP system (table 1 in the supplementary data) generates recommendations from the very strong (unequivocal benefit/risk ratio, high-quality evidence; grade 1A) to the very weak (questionable benefit/risk ratio, low-quality evidence; grade $2 \mathrm{C}$ ). Each recommendation was voted by all experts: if $<85 \%$ of the experts were in total agreement with one proposal, the corresponding recommendation was modified after a new discussion. These recommendations are detailed later in the guidelines.

It should be noted that the authors of the ACCP recommendations also stated: "whatever the grade of the recommendation, clinicians must use their judgment, considering both local and individual patient circumstances, and patient values, in making individual decisions. In general, however, they should place progressively greater weight on expert recommendations as they move from grade $2 \mathrm{C}$ to grade $1 \mathrm{~A} . "$ [8]. This explains why the ERS/ESTS experts have used different terms in their recommendations ("should" or "may" for example) to modulate the strength of each recommendation to the reader in the clinical practice.

\section{EPIDEMIOLOGY OF MALIGNANT MESOTHELIOMA What are the risk factors associated with MPM?}

\section{Asbestos}

Asbestos is the principal aetiological agent of MPM. This term refers to a group of six silicate minerals which are able to form very thin fibres: chrysotile, crocidolite, amosite, anthophyllite, tremolite and actinolite. Chrysotile belongs to the serpentine group and the others to the amphibole group of minerals. Chrysotile is less biopersistent in the lungs than amphiboles. Chrysotile, amosite and crocidolite have all been widely used for industrial purposes.

The first studies on the association between asbestos and MPM were published in the 1960s [9]. As most asbestos exposure is work-related, mesothelioma is an occupational disease in the majority of cases. The background incidence is very low. Because past exposure to asbestos was more common in occupations with a predominantly male workforce, the current incidence of MPM is higher among males than females. For example, according to the French National Mesothelioma Surveillance Program, the risk fraction attributable to occupational asbestos exposure is $>80 \%$ in males and $<40 \%$ in 
females [10]. This sex difference in risk fraction attributable to occupational exposure to asbestos has also been reported in other countries.

Over the last decades, a shift has been observed in the exposure history of mesothelioma cases, from primary asbestos workers (handling raw asbestos material) to end-users often exposed when installing asbestos products or handling asbestos materials that are still in place, e.g. construction workers, electricians, plumbers and heating workers. Even if the occupations with the highest risk of mesothelioma belong to the first group, the number of subjects at risk of MPM is presently much larger in the latter group.

Environmental mesotheliomas are either linked to "natural" exposure in areas of the world where asbestos (generally tremolite) exists as a geological component of the soil (Turkey, Corsica, Cyprus and New Caledonia) or where it is often used for white-washing walls of houses, or to neighbourhood exposures in people living close to asbestos mines or factories $[11,12]$. Para-occupational cases are described in households of asbestos workers, mainly because of domestic exposure via clothes used at work.

A dose-effect relationship has been demonstrated, but it is impossible to define a threshold of cumulative exposure below which there is no increased risk [13]. Therefore, all individuals who have been exposed to asbestos are considered to be a population at risk. The mean (range) latency of MPM after exposure to asbestos is $\sim 40(15-67)$ yrs. In a review of 1,690 cases, the latent period was $>15$ yrs in $99 \%$ of cases [14].

Among commercially used fibres, crocidolite and amosite have a higher carcinogenic pleural potency than chrysotile fibres. The carcinogenic potency of short asbestos fibres cannot be ruled out at present time.

MPM may be observed in exposed individuals without any other asbestos-related disease (lung or pleural fibrosis). In most cases, pleural plaques are a sign of asbestos exposure in the past, and it has been reported that they are associated with a greater risk of mesothelioma. Indeed, it is expected that mesothelioma is more frequent in subjects having had pleural plaques than in the general population because both diseases are strongly associated with asbestos exposure. Such association has been reported in some necropsy or cohort studies. In contrast, other cohort studies did not report such an association. In a cancer prevention programme at the crocidolite mining and milling town of Wittenoom (Australia), pleural thickening was not associated with an increased risk of pleural mesothelioma after adjusting for time since first exposure, cumulative exposure and age at the start of the programme [15]. The same authors reported an excess of peritoneal mesothelioma in this population [15]. Therefore, overall there is no clear evidence that pleural plaques alone increase the risk of pleural mesothelioma.

\section{Evidence}

The global attributable proportion of MPM to asbestos is $>80 \%$ in males but much less in females. A dose-response relationship is clearly established for asbestos and MPM, but the disease may be observed in subjects having low-dose cumulative exposures. MPM is mainly observed following asbestos exposure from occupational origin, but it is also observed in para-occupational and environmental exposures to asbestos. Most amphibole fibres, particularly crocidolite but also amosite and tremolite, have a higher carcinogenic pleural potency than chrysotile fibres. Most workers have experienced mixed exposure to various asbestos types. Mesothelioma has been associated with chrysotile exposure, but in most cases chrysotile was contaminated or associated with amphibole fibres. At present, the carcinogenic potency of short asbestos fibres cannot be ruled out. In most cases, pleural plaques are a sign of previous asbestos exposure. There is no clear evidence that pleural plaques alone would increase the risk of MPM. MPM may be observed in exposed individuals without any other asbestosrelated disease.

\section{Statement}

The low proportion of MPM attributable to asbestos in females is not yet fully understood and merits further investigations, including investigations for occult asbestos exposure and/or for other aetiological factors (grade 2B).

\section{Other factors}

Agents other than asbestos are considered to be recognised or potential risk factors or cofactors for MPM, namely exposure to other natural (erionite and fluoro-edenite) or man-made (refractory ceramic) fibres, ionising radiation and SV 40 virus. It is known that tobacco does not play a role in the development of mesothelioma. From the available published data, there is no evidence of pleural carcinogenic potency of man-made (vitreous) fibres, such as mineral wool (rockwool, glasswool and slagwool) fibres in humans. Genetic factors, which could increase susceptibility, may contribute to the development of MPM, consistently with familial clusters of mesothelioma. One study has suggested that genetic predisposition influences mineral fibre carcinogenesis in Karain (Turkey) where erionite is implicated in an extremely high incidence of the disease $[16,17]$.

\section{Evidence}

For some agents, the level of evidence is highly in favour of a causative role in MPM: erionite and therapeutic irradiation (e.g. for breast cancer or lymphoma). For other agents or situations, there is still controversy or a lower level of evidence in humans: refractory ceramic fibres and SV40 virus. From the available published data, there is no evidence of pleural carcinogenic potency of mineral wool (rockwool, glasswool and slagwool) fibres in humans. Tobacco smoking is not carcinogenic to the pleura.

\section{What are the future trends in the epidemiology of MPM?}

There are prominent differences in incidences reported from different countries worldwide [18]. The incidences vary from 7 per million (Japan) to 40 per million (Australia) inhabitants per year [19]. In Europe, the incidence is $\sim 20$ per million. It is reasonable to accept that these differences are mainly due to differences in historical asbestos import and consumption but an influence of diagnostic practices and awareness may also interfere.

In the future, epidemiologists expect peak incidences in the very next decades. Preliminary projections from the 1990s were recently re-evaluated and the date of peak incidence and 
number of cases were generally less than previously anticipated [2, 20-22]. Peak incidence is expected between 2015 and 2020 in Europe [19], and may already have been reached in some countries (USA and Sweden).

\section{Evidence}

There are differences in MPM incidence between countries, which mainly reflect differences in asbestos consumption over the past decades in these countries. Because of the long latency of MPM and the national differences in the timing of reduction or ban of asbestos use, the timing of the peak incidence of MPM cannot be predicted precisely and may vary from one country to another. Epidemiological projections have suggested that the incidence of MPM could still increase in Europe for the next 10 yrs. In countries that continue to use asbestos in the 21st century, the incidence of MPM is expected to increase in the forthcoming decades.

\section{What are the available methods to evaluate exposure to asbestos?}

Several methods and tools exist to evaluate cumulative exposures, such as occupational questionnaires and the use of job/exposure matrices. Due to the long latency period of the disease and the lack of precise data on airborne fibre levels, the exact evaluation may be difficult, especially for people other than experienced occupational hygienists or occupational physicians.

Mineral analyses (MA) of biological samples (bronchoalveolar lavage (BAL) and lung tissue) by light or electron microscopy can provide information about the retained asbestos dose, mainly for amphiboles which have a longer pulmonary biopersistence than chrysotile. Due to the long latency period of MPM and the fact that MPM can be associated with low-dose exposures, MA will not always show high levels of asbestos fibres or bodies. However, they may be useful in revealing high levels of fibres when exposure history is unknown or difficult to assess (e.g. indirect exposures). They may also identify specific environmental fibres (e.g. tremolite) [23].

Most MPM cases are linked to past occupational exposure, and MPM is recognised as an occupational disease in most, if not all, national worker's compensation schemes. As MPM is generally a severe and fatal disease, the social security aspects are important for the patient and the relatives. As with other occupational cancers, mesotheliomas are under-reported. It is advisable to systematically assess the past exposure history of MPM patients according to the practices of the national worker's compensation or other relevant social security schemes [10].

\section{Evidence}

Evaluation of asbestos exposure in a patient with MPM can be made with different tools, mainly through specific occupational and environmental questionnaires.

\section{Recommendation}

Evaluation of asbestos exposure (mainly through specific occupational and environmental questionnaires) is relevant and should be performed for social security and medico-legal purposes according to relevant national practices (grade 1A).

\section{Statement}

Exposure assessment is also important in specific scientific purposes. However, it has no therapeutic relevance and may be difficult to perform without the help of occupational hygienists or occupational physicians (experts' advice).

The above principles also apply for mineralogical analysis of biological samples (quantification of asbestos bodies or asbestos fibres in BAL fluid or lung tissue samples). Such mineralogical analyses are not required in the clinical management of mesothelioma.

\section{Is there a rationale for MPM screening?}

A screening programme is medically justified if the detection of the disease at an earlier stage improves the prognosis by more effective medical or surgical treatment. To date, according to the data available on MPM (prevalence, prognosis and treatment) and the performance (sensitivity, specificity) of potential screening methods, the medical efficacy of a largescale screening is not established $[4,5]$.

Low-dose computed tomography (CT) scan has not been proven to be an effective screening tool for the detection of early MPM: no single case of pleural mesothelioma was detected in a cohort of 1,045 asbestos exposed workers [24]. Positron emission tomography (PET) scan and magnetic resonance imaging (MRI) are imaging techniques that are useful in the clinical management of malignant pleural diseases and in the differentiation of malignant from benign pleural disease, but are not available and/or applicable for screening purposes.

Biological markers, such as soluble mesothelin related peptides, soluble mesothelin related peptides (SMRP) and osteopontin, are currently studied [25]. Because of the sensitivity and specificity of available biological markers, and because of the prevalence of the disease, the number of false-positive tests would be several times higher than true-positive subjects identified if screening was proposed to all asbestos-exposed subjects. Therefore, biological markers cannot be presently proposed as screening tools $[4,5]$. A recent study assessed the value of serum SMRP as a screening test. In a prospective test in 538 individuals with an occupational exposure to asbestos, a low specificity and high number of false-positive values were found [26]. No mesothelioma was observed but one lung cancer and one suspected cardiac tumour were observed in this cohort, although 15 (almost 3\%) subjects had elevated SMRP levels. This fact could result in a large number of patients needing to be followed up with expensive, and possibly harmful, investigations for many years [27]. Finally, there is no proof that early discovery of MPM will cure the patient or even improve their survival for many months. The authors concluded that SMRP tests should not be used for screening pending the results of ongoing large prospective studies that not only examine its diagnostic accuracy but also the relationship between SMRP levels and survival-specific and disease-specific mortality [26].

\section{Recommendations}

To our knowledge, there is no place for screening of MPM (grade 1B). The usefulness of thoracic imaging and/or biological markers should be further evaluated in selected 
highly exposed populations included in voluntary surveillance protocols (grade 1B).

\section{DIAGNOSIS OF MPM}

\section{From a clinical point of view}

Are there any diagnostic clinical criteria?

Recommendation

The clinical manifestations of MPM are usually nonspecific and insidious and should not be used alone as diagnostic criteria, even in case of previous asbestos exposure (grade 1A).

\section{Are there any specific diagnostic imaging criteria?} Recommendations

Chest radiographs usually show a unilateral pleural effusion or thickening. Chest radiographs alone should not be used for the diagnosis of MPM (grade 1A) [28].

A chest CT scan is unsuitable for definitive diagnosis of MPM, but diffuse or nodular pleural thickening are suggestive of the disease (grade 1A) [28, 29].

\section{Statement}

MRI is not relevant for the diagnosis of mesothelioma (grade 1B) [29]. PET scanning is currently not useful for the diagnosis of mesothelioma (grade 1C) [29-31].

\section{What is the role of thoracoscopy for the diagnosis?}

When a mesothelioma is suspected on clinical or radiological data, thoracoscopy is the best method to obtain the diagnosis. For more information refer to the Pathology section.

\section{Recommendations}

It is recommended, except in the case of pre-operative contraindication or pleural symphysis, to perform thoracoscopy for the diagnosis of MPM (grade 1A).

\section{From a pathological point of view}

The accurate diagnosis of mesothelioma, a malignant tumour that arises from mesothelial cells that line the serosal cavities, is made on histopathological examination. However, diagnosis can be difficult because mesothelioma is a very heterogeneous cancer which creates various misleading histopathological pitfalls. Moreover, the pleura is a common site for metastatic disease.

The macroscopic aspect of mesothelioma may vary during its natural history, thus it depends when the mesothelioma is first observed. As pleural mesotheliomas progress, their gross appearance becomes more suggestive of MPM to some extent, although other malignant tumours may have a pseudomesotheliomatous aspect (thymomas, carcinomas, lymphomas, angiosarcomas, etc.). The microscopic characteristics of MPM are well defined in the new international classification of pleural tumours [32]. However, this tumour has a varied and deceptive appearance in a high percentage of cases and may resemble benign pleural lesions or metastatic lesions, which are much more common than mesothelioma in the general population. Thus, the most frequent metastatic pleural tumours are from lung or breast carcinoma (in $7-15 \%$ and $7-11 \%$, respectively) whose morphology can be mistaken for mesothelioma on standard sections stained with haematoxylineosin-saffron. Diagnostic problems also occur with frequent benign inflammatory or reactive lesions of the pleura that may occur in patients at approximately the same age as in MPM (pleural effusion during cardiac failure, collagen disease, pneumonia, digestive disease such as cirrhosis, etc.). These lesions are often secondary and lead to atypical mesothelial hyperplasia which can result in diagnostic error. In a validation exercise carried out in France by the Pathology Group for Assistance in the Diagnosis of Mesothelioma, within the context of the National Program of Mesothelioma Survey (1998-2007), such errors represent $13 \%$ of initially diagnosed cases [10].

Which specimens for which clinical presentation?

As pleural effusion is usually the first clinical sign of MPM, cytology is often the first diagnostic examination to be carried out.

\section{Recommendations}

It is not recommended to make a diagnosis of mesothelioma based on cytology alone because of the high risk of diagnostic error (grade 1B).

It is recommended that a cytological suspicion of mesothelioma is followed by tissue confirmation (grade 1B).

Disease recurrence and metastases can be ascertained on cytology alone. This recommendation is in agreement with that proposed by the International Mesothelioma Panel (grade 1B).

Diagnosis of mesothelioma from fine needle biopsies (Abrams or Castelain needles) is associated with the same problems as cytology. A conclusive diagnosis can only be made if the material is representative of the tumour, in sufficient quantity to allow immunohistochemical characterisation and in the context of appropriate clinical, radiological and/or surgical findings.

\section{Recommendations}

Thoracoscopy should be preferred for diagnostic investigation, allowing complete visual examination of the pleura, multiple, deep and large biopsies (preferably including fat and/or muscle to assess tumour invasion) and providing a diagnosis in $>90 \%$ of cases (grade $1 \mathrm{~A}$ ).

Fine needle biopsies are not primarily recommended for the diagnosis of mesothelioma because they are associated with low sensitivity ( 30\%) (grade $1 \mathrm{~A})$.

It is recommended to take biopsies of both normal and seemingly abnormal pleura (grade 1C). It is not recommended to make a diagnosis of MPM solely on frozen tissue sections (grade 1B).

What classification should be used?

Recommendations

It is recommended that the WHO 2004 classification [32] be used for mesothelial tumours (grade 1A). This provides a comparative basis for diagnosis, prognosis and patient management. An updated classification from the International Mesothelioma Interest Group is expected in 2009.

Should a complementary immunohistochemical examination be carried out in addition to morphological examination, and which immunohistochemical markers and how many antibodies should be used for which histological variants?

Recommendations

It is recommended that a diagnosis of MPM always be based on immunohistochemical examination (grade 1A). 
The International Mesothelioma Panel has put forward various recommendations. The immunohistochemical approach depends on whether the tumour subtype of mesothelioma is epithelioid or sarcomatoid.

\section{Recommendations}

To separate epithelioid mesothelioma from adenocarcinoma, it is recommended that two markers with positive diagnostic value for mesothelioma (nuclear markers such as anticalretinin and anti-Wilms tumour antigen-1 or the membrane marker anti-epithelial membrane antigen (EMA); for epithelioid mesothelioma, anti-cytokeratin(CK)5/6, antiD2-40 (podoplanin) or anti-mesothelin, etc.) and two markers with negative diagnostic value (anti-Ber-EP4, a membrane marker; antithyroid transcription factor-1, a nuclear marker; or monoclonal anti-carcinoembryonic antigen, anti-B72-3, anti-MOC-31, antioestrogen/progesterone, anti-EMA, cytoplasmic staining) are used to validate the diagnosis (grade 1A). Among the various sources of antibodies, it is mandatory to use those presenting at a minimum of $60-70 \%$ sensitivity. It is not recommended to use anti-CK7/anti-CK20 to make the diagnosis of mesothelioma (grade 1A). The antibodies requirements are summarised in table 1 .

To separate sarcomatoid mesothelioma from squamous and transitional cell carcinoma (table 2), it is recommended to use two broad-spectrum anti-cytokeratin antibodies and two markers with negative predictive value (such as anti-CD34 and anti-B-cell lymphoma 2 marker, anti-desmin, anti-S100) to confirm the diagnosis (grade 1A). Negative immunostaining with a single antibody does not exclude the diagnosis (grade 1C).

With regard to atypical mesothelial hyperplasia (superficial mesothelial proliferations), there are currently no commercially available immunohistochemical markers that identify the benign or malignant nature of the cells observed.

\section{Should electron microscopic examination and molecular biology be performed? \\ Recommendations}

Electron microscopy and molecular biology should not be carried out routinely to confirm the diagnosis of mesothelioma (grade 1A).

\section{Statement}

There are no diagnostic or therapeutic reasons for freezing pleural tumour tissue (grade 1A).

\section{Should the advice of an expert panel be sought faced with a suspicion of MPM?}

Recommendations

An independent expert panel should be asked to confirm the diagnosis particularly in clinical trials, or in any case where there is doubt about the diagnosis (grade 1B).

TABLE 1 Immunohistochemistry to separate epithelioid mesothelioma from adenocarcinoma

\begin{tabular}{|c|c|c|c|c|c|}
\hline Antibody & Current value & Mesothelioma & Positivity & Adenocarcinoma & Positivity \\
\hline \multicolumn{6}{|l|}{ Mesothelioma } \\
\hline Calretinin & Essential & $\begin{array}{c}\text { Positive } \\
\text { (nuclear and cytoplasmic) }\end{array}$ & $80-100 \%$ & Usually negative & $\begin{array}{c}\text { 5-10\% cytoplasmic } \\
\text { positivity of lung } \\
\text { adenocarcinoma }\end{array}$ \\
\hline Keratin CK5/6 & Useful & $\begin{array}{c}\text { Positive } \\
\text { (cytoplasmic) }\end{array}$ & $60-100 \%$ & Usually negative & $2-10 \%$ focal positivity \\
\hline WT-1 & Useful & $\begin{array}{l}\text { Positive } \\
\text { (nuclear) }\end{array}$ & $43-93 \%$ & $\begin{array}{c}\text { Lung adenocarcinoma are } \\
\text { negative }\end{array}$ & $0 \%$ \\
\hline EMA & Useful & $\begin{array}{c}\text { Positive } \\
\text { (membranous) }\end{array}$ & $60-100 \%$ & Positive (cytoplasmic) & $70-100 \%$ \\
\hline Podoplanin & Useful & $\begin{array}{c}\text { Positive } \\
\text { (membranous) }\end{array}$ & $80-100 \%$ & Usually negative & $7 \%$ focal positivity \\
\hline \multicolumn{6}{|c|}{ Lung adenocarcinoma } \\
\hline CEA monoclonal & Very useful & Almost invariably negative & $0 \%$ & Positive (cytoplasmic) & $50-90 \%$ \\
\hline CD15 & Useful & Never expressed in mesothelioma & $0 \%$ & Positive (membranous) & 50-70\% focally positive \\
\hline Ber-EP4 & Very useful & $\begin{array}{l}\text { Positive or negative } \\
\text { (membranous) }\end{array}$ & $\begin{array}{l}\text { Up to } 20 \% \text { can be } \\
\text { focally positive }\end{array}$ & Positive (membranous) & $95-100 \%$ \\
\hline TTF-1 & Very useful & Never expressed & $0 \%$ & Positive (nuclear) & $\begin{array}{l}70-85 \% \text { of lung } \\
\text { adenocarcinoma }\end{array}$ \\
\hline B72.3 & Very useful & Rarely positive & $<1 \%$ & Positive (cytoplasmic) & $\begin{array}{l}70-85 \% \text { of lung } \\
\text { adenocarcinoma }\end{array}$ \\
\hline \multicolumn{6}{|l|}{ Breast carcinoma } \\
\hline ER & Very useful & Never expressed in mesothelioma & $0 \%$ & Positive nuclear staining & $\sim 70 \%$ \\
\hline
\end{tabular}

CK5/6: cytokeratin 5/6; WT-1: Wilms tumour antigen-1; EMA: epithelial membrane antigen; CEA: carcinoembryonic antigen; TTF-1: thyroid transcription factor-1; ER: endoplasmic reticulum marker. 
TABLE 2 Immunohistochemistry for separating sarcomatoid mesothelioma from squamous and transitional cell carcinoma

\begin{tabular}{|c|c|c|c|c|c|}
\hline \multicolumn{6}{|l|}{ Mesothelioma } \\
\hline Calretinin & Useful & $\begin{array}{c}\text { Positive (strong nuclear and } \\
\text { cytoplasmic) }\end{array}$ & $80-100 \%$ & Usually cytoplasmic positivity & $5-40 \%$ \\
\hline Keratin CK5/6 & Not useful & Positive (cytoplasmic) & $60-100 \%$ & Cytoplasmic positivity & $100 \%$ \\
\hline p63 & Very useful & Almost always negative & $0 \%$ & Positive (nuclear) & $\sim 100 \%$ \\
\hline Ber-EP4 & Useful & Positive or negative & Up to $20 \%$ are positive & Positive (cytoplasmic) & $80-100 \%$ \\
\hline MOC 31 & Useful & $\begin{array}{l}\text { Positive or negative (focal } \\
\text { membranous staining) }\end{array}$ & $2-10 \%$ & Positive (membranous) & $97-100 \%$ \\
\hline
\end{tabular}

CK5/6: cytokeratin 5/6; WT-1: Wilms tumour antigen-1.

\section{STAGING, PRE-THERAPEUTIC INVESTIGATIONS AND PROGNOSTIC FACTORS}

\section{Which staging classification is used?}

Staging describes the anatomical extent of a tumour. There are at least five staging systems available in pleural mesothelioma, the latest one devised by members of the International Mesothelioma Interest Group and approved by the Union International Contre le Cancer (UICC) (table 2 in the supplementary data) [33]. The main drawback of the classifications is the inaccuracy in describing $\mathrm{T}$ - and N-extent by current imaging techniques. Because of this, an international panel of experts could not agree on a common staging classification in pleural mesothelioma and strongly advocated the development of a new robust and uniform clinical staging system that should be prospectively validated, TNM-based and include the existing surgical-pathological staging systems.

\section{Recommendations}

In the absence of a uniform, robust and validated staging system, the experts advocate the use of the most recent TNMbased UICC classification (grade 1C) [33].

What are the minimal pre-treatment staging examinations?

The following assumptions were made by the experts' panel: 1) an optimal pre-treatment assessment protocol should be simple and widely applicable, sequential and logical, not unnecessarily invasive and identify candidates for proper treatment; 2) the functional and psychological suitability of individual patients for different forms of therapy should be assessed separately (i.e. cardiac and/or pulmonary function); and 3) a profound assessment of asbestos exposure should be made in every patient at presentation and recorded in the medical file.

\section{Evidence}

The pre-treatment assessment is empirically split into three steps which are, to some degree, overlapping [34]. Whether a patient goes through all three steps strongly depends on the results of the procedures and the consequences for the choice of treatment with radical or palliative intent only.
Step I is to be considered in all patients at presentation or diagnosis (table 3). Step II is to be considered in patients who are candidates for any kind of active treatment (table 4). Step III is the final process of patient selection for combined modality or radical locoregional treatment (table 5). It is the opinion of the experts that this last step will only considered in a minority of patients with pleural mesothelioma. This is reflected in the paucity of evidence, reflecting different institutional practice. Among the investigations to be considered are mediastinoscopy, MRI of the chest, video-assisted thoracoscopy (VATS), enobronchial ultrasound-fine needle aspiration (E(B)US-FNA), FDG-PET scan and laparoscopy. In the absence of comparative trials, no formal advice regarding their respective efficacy can be given

The experts further agree that in patients proceeding to step II or higher: 1) a diagnosis of mesothelioma should be confidently established, preferably on a biopsy specimen with adequate immunohistochemistry and subtyping; 2 ) the interval within which the pre-treatment assessment has to be finalised should be as short as possible; and 3 ) recent ( $<1$ month) imaging studies should be available prior to invasive procedures. Further research should be performed with regard to the comparative efficacy of different intrathoracic techniques (mediastinoscopy, VATS, and EUS-FNA) and the value of the new techniques (PET-CT, EBUS-FNA).

\section{Recommendations}

A three-step pre-treatment assessment is recommended based on empirical observation, good clinical practice and the fact that the treatment intent differs between patients (grade 1C).

\section{Which prognostic factors are of importance?}

Prognostic factors are pre-treatment clinical or biological characteristics of patient or tumour which impact on the outcome, regardless of the treatment installed.

\section{Evidence}

Several prognostic factors have been described in large multicenter series and have been independently validated [35]. Among these, the Surveillance, Epidemiology and End Results (SEER) Program review is a landmark retrospective 
TABLE 3 Parameters to be considered in all patients at presentation/diagnosis

\begin{tabular}{lcc} 
Investigations & Including & Confirmatory tests \\
\hline $\begin{array}{l}\text { Demographics } \\
\text { Clinical history }\end{array}$ & $\begin{array}{c}\text { Sex and age, asbestos exposure } \\
\text { Performance status, comorbidities, presence/absence of } \\
\text { chest pain, dyspnoea, change in body weight or BMl } \\
\text { Presence or absence of "shrinking hemithorax", } \\
\text { cutaneous nodules } \\
\text { Chest radiograph, PA/lateral }\end{array}$ & As appropriate \\
Radiological investigations & $\begin{array}{c}\text { Chest radiograph, inspiration/expiration, } \\
\text { pre-/post-drainage of pleural fluid }\end{array}$ \\
\hline Blood tests & Haemoglobin, leukocytes, platelets, basic biochemistry
\end{tabular}

BMI: body mass index; PA: postero-anterior.

series of 1,475 patients with histological confirmed mesothelioma and showing that age, sex, tumour stage, treatment and geographic area of residence were important prognostic factors [36]. A number of factors, such as performance status, stage and weight loss, are common to other tumours; others factors, such as age and sex have not been confirmed in all series. Symptoms and quality of life are increasingly being investigated as prognostic factors. Nonepitheloid subtype is consistently associated with a poorer prognosis. Of the numerous biological factors studied, low haemoglobin level, high lactose dehydrogenase (LDH), a high white blood cell count and a high thrombocyte count have been repeatedly associated with a poor prognosis. New serum biomarkers with potential prognosis significance (e.g. soluble mesothelin and osteopontin) are currently under investigation [37-39]. Based on these various factors, three prognostic scores have been developed and prospectively validated; the CALGB (Cancer and Leukaemia Group B) and two EORTC (European Organization for Research and Treatment of Cancer) prognostic scoring systems (table 6) [40,41]. The latter was later adapted according to the results of the multivariate analysis of prognostic factors of a large randomised chemotherapy trial in good performance patients $[42,43]$.

\section{Recommendations}

Performance status of the patient and histopathological subtype are currently the only prognostic factors of clinical importance that may routinely be used in the management of patients with malignant mesothelioma (grade 2A).
Other parameters with prognostic capacity, such as age, sex, stage, presence or absence of certain symptoms and haematological factors, should be recorded at baseline and reported in clinical trials (grade 2A).

\section{TREATMENT OF MPM}

\section{Surgery for MPM}

What is the evidence for debulking decortication/pleurectomy for symptom control?

Debulking pleurectomy/decortication can be defined as significant but incomplete macroscopic clearance of pleural tumour. The objective of the operation is to relieve an entrapped lung by removing the visceral tumour cortex. Removal of the parietal tumour cortex may relieve a restrictive ventilatory deficit and reduce chest wall pain. The operative procedure may be performed by either open thoracotomy or closed VATS.

\section{Evidence}

There is limited evidence supporting debulking surgery. At present there is an absence of randomised trials, but a national study is ongoing in the UK which is being supported by the National Cancer Research Institute comparing VATS debulking with chemical pleurodesis (MesoVATS). There are a small series of retrospective studies which provide low-grade evidence for debulking pleurectomy [44-47]. The associated morbidity of thoracotomy may diminish the benefits [48]; however there is limited but emerging evidence that VATS can provide good symptom control and may have a beneficial effect on survival [46].

TABLE 4 Investigations performed in patients likely to receive some form of active treatment

\begin{tabular}{|c|c|c|}
\hline Investigations & Including & Confirmatory tests \\
\hline Primary tumour & Adequate biopsy for histology confirmation & \\
\hline CT scan of chest and upper abdomen & $\begin{array}{l}\text { Spiral technique, with i.v. contrast, including at least level of both } \\
\text { kidneys after drainage of pleural fluid }\end{array}$ & \\
\hline Pulmonary function tests & Forced vital capacity, forced expiratory volume in $1 \mathrm{~s}$ & \\
\hline Bone scan & Not routine, to be considered on clinical suspicion only & $\mathrm{CT} / \mathrm{MRI}$ to confirm dubious findings \\
\hline Brain CT/MRI & Not routine, to be considered on clinical suspicion only & \\
\hline
\end{tabular}


TABLE 5 Investigations to be considered in patients who are candidates for surgery or multimodal treatment

\begin{tabular}{|c|c|c|c|}
\hline Pulmonary function tests & $D \mathrm{~L}, \mathrm{CO}$ in addition to $\mathrm{FVC}$ and $\mathrm{FEV} 1$ & $\begin{array}{l}\text { Assessment similar to the one } \\
\text { for lung cancer }\end{array}$ & $\begin{array}{l}\text { Lung scintigraphy probably performed as } \\
\text { for any pulmectomy }\end{array}$ \\
\hline Primary tumour & Adequate biopsy for histological subtyping & & \\
\hline Diaphragm & CT scan or MRI & & \\
\hline Extra-thoracic, to exclude "occult" M1 & $\begin{array}{l}\text { FDG-PET/CT } \\
\text { Laparoscopy }\end{array}$ & According to institutional practice & Biopsy of suspected extrathoracic lesions \\
\hline
\end{tabular}

$\mathrm{LL}, \mathrm{CO}$ : diffusing capacity of the lung for carbon monoxide; FVC; forced vital capacity; FEV1: forced expiratory volume in $1 \mathrm{~s}$; CT: computed tomography; MRI: magnetic resonance imaging; FDG-PET: 18-Fluor-2-deoxy-glucose positron emission tomography; VATS: video-assisted thoracic surgery; E(B)US-FNA: enobronchial ultrasoundfine needle aspiration.

\section{Recommendations}

Pleurectomy/decortication should not be proposed in a curative intent but can be considered in patients to obtain symptom control, especially symptomatic patients with entrapped lung syndrome who cannot benefit from chemical pleurodesis (grade 2C). The VATS approach is preferred (grade 1C).

What is the evidence for radical surgery in MPM?

Radical surgery may be defined as an attempt to remove all macroscopic tumours from the hemithorax. These objectives are usually achieved by extrapleural pneumonectomy (EPP) with en bloc resection of pleura, lung, pericardium and diaphragm and systematic nodal dissection.

\section{Evidence}

There is limited evidence for the efficacy of radical surgery for mesothelioma. Among resected mesothelioma patients, the only published long-term survivors have undergone radical surgery (EPP) as part of a multimodality programme [49-51]. There have been a number of subsequent prospective and retrospective series which have all demonstrated a similar median survival of 20-24 months [49-51]. Operative mortality has fallen to an acceptable level of $\sim 5 \%$ in experienced centres [51] but morbidity remains high at $\sim 50 \%$.

\section{Recommendations}

Radical surgery (EPP) should only be performed in clinical trials, in specialised centres, as part of multimodal treatment.

TABLE 6 Prognostic scoring systems in malignant mesothelioma

\begin{tabular}{|c|c|c|c|c|c|}
\hline \multirow[t]{4}{*}{ CALGB } & HeRnDON [40] & 337 & Performance status & Good & Poor \\
\hline & & & Chest pain & Absent & Present \\
\hline & & & Platelet count & $<400 \times 10^{12} \cdot \mathrm{L}^{-1}$ & $\geqslant 400 \times 10^{12} \cdot \mathrm{L}^{-1}$ \\
\hline & & & LDH & $<500 \mathrm{IU} \cdot \mathrm{L}^{-1}$ & $\geqslant 500 \mathrm{IU} \cdot \mathrm{L}^{-1}$ \\
\hline \multirow{3}{*}{ EORTC } & & & Sex & Female & Male \\
\hline & & & Certainty of diagnosis & Definite & Possible \\
\hline & & & WBC count & $<8.3 \times 10^{9} \cdot \mathrm{L}^{-1}$ & $\geqslant 8.3 \times 10^{9} \cdot \mathrm{L}^{-1}$ \\
\hline \multirow[t]{3}{*}{ EORTC\# } & VAN MeERBEECK [42] & 250 & Stage & $1-||$ & III-IV \\
\hline & & & Histology & Epitheloid & Nonepitheloid \\
\hline & & & Appetite loss & Absent & Present \\
\hline
\end{tabular}

CALGB: Cancer and Leukaemia Group B; EORTC: European Organization for Research and Treatment of Cancer; LDH: lactate dehydrogenase; WBC: white blood cell.

\#: performance status 0-1 was an inclusion criterion for this series; ": difference between actual value and $16 \mathrm{~g} \cdot \mathrm{dL}^{-1}$ and $14 \mathrm{~g} \cdot \mathrm{dL}^{-1}$ in males and females, respectively. 


\section{Radiotherapy in MPM}

What is the role of "palliative" radiotherapy aimed at pain relief? Recommendations

Palliative radiotherapy aimed at pain relief may be considered in cases of painful chest wall infiltration or nodules (grade 2C).

What is the role of radiotherapy in the prevention of parietal seeding along the drainage channels?

BOUTIN et al. [52] previously suggested that an irradiation with $3 \times 7$ Gy for 3 consecutive days in the 4 weeks following drainage or thoracoscopy should be performed to prevent subcutaneous metastasis developing along drainage channels or thoracocentis tracts. However, a recent randomised trial was published comparing immediate drain site radiotherapy $21 \mathrm{~Gy}$ in three fractions to best supportive care in 61 patients treated between 1998 and 2004, with no difference in terms of tract metastatic recurrence between the two arms $[53,54]$. O'ROURKE et al. [53] concluded that prophylactic drain site radiotherapy in MPM did not reduce the incidence of tumour seeding as indicated in previous studies and reached the same conclusion as CHAPMAN et al. [55]. Suboptimal techniques of radiotherapy may explain the discrepancy of these results, and should certainly be an issue.

\section{Recommendations}

The value of prophylactic radiotherapy is questionable. Therefore, the experts were not able to draw any recommendation.

What is the role of post-operative radiotherapy?

Data from the literature are limited and come from retrospective studies.

\section{Recommendations}

Radiotherapy should not be performed after pleurectomy or decortication (grade 1A). Post-operative irradiation after EPP should only be proposed in clinical trials, in specialised centres, as a part of multimodal treatment (grade 1A).

In the absence of phase III randomised trials, the establishment of a prospective controlled study evaluating the efficacy and tolerability of adjuvant radiotherapy post-EPP (minimum dose of $50 \mathrm{~Gy}$ with daily fraction size of 1.8 to $2 \mathrm{~Gy}$ ) is recommended (grade 1C). A randomised multicenter European study is ongoing to answer this question (SAKK study). Retrospective studies seem to show a radiation dose effect that should be further studied with conformal radiation technique. In the study by Rusch et al. [50], who used 54 Gy hemithorax radiation as adjuvant therapy after EPP, the local recurrence rate was $13 \%$ with a $4 \%$ local-only recurrence rate, whereas in the study by BALDINI [56], there was a 50\% local recurrence rate with a $13 \%$ local-only rate after trimodality therapy. The ability to fully cover all the areas at risk, limited by the surrounding normal structures (heart and liver, particularly), the total dose given and radiotherapy technique contribute to explain these discrepancies.

\section{What is the place for intensity-modulated radiotherapy in MPM after EPP?}

Preliminary results of intensity-modulated radiotherapy in the adjuvant setting after EPP seem particularly promising as they provide good local control and protect organs at risk, such as the heart or liver. However, severe pulmonary toxicity has been reported in recent studies so it should not be recommended outside of clinical trials; six out of 13 patients developed fatal pneumonitis [57].

Derived from these recent retrospective studies, so as to predict the risk of pneumonitis, the following pulmonary dosimetric values (V20, V5 and mean lung dose) should be specified. The V20 (volume of both lung minus the planned target volume (PTV)) should be $<15 \%$, and the mean lung dose should be $<10 \mathrm{~Gy}$. These dosimetric constraints can be used for conformal radiotherapy as well. The dose-volume histograms (DVH) of all target volumes (clinical target volume and PTV) and of all critical organs (contralateral lung, cardiac volume, spinal cord, oesophagus, liver, and right and left kidney) should be clearly stated.

\section{Statement}

Further studies are needed to better establish the role of radiotherapy. Recent studies have underlined the importance of radiotherapy technique both in terms of local control and toxicity.

\section{Recommendations}

Therefore, it is recommended to carry out this radiotherapy in specialised centres only (advice of experts).

\section{Chemotherapy of MPM}

The methodology used to answer the following questions has been previously described $[4,58]$. The recommendations were based on: 1) the on-line recommendations from Cancer Care Ontario [59]; 2) the literature review with meta-analysis published by BERGHMANS et al. [60], which was updated in 2003 [61], completed by the articles published after these reviews until January 2009; and 3) the French recommendations on chemotherapy in MPM published by SPLF $[4,58]$.

\section{Has the benefit of chemotherapy been demonstrated?}

Currently, only one randomised study assessed the efficacy of chemotherapy versus placebo in malignant mesothelioma. Results were presented at the American Society of Clinical Oncology and European Crohn's and Colitis Organisation in 2007 [62]. No survival difference was observed between both arms, excepted for a trend favouring the vinorelbine subgroup. It must be pointed that, according to the results of randomised studies and the systematic review [60, 61], the choice of comparative chemotherapy was probably not adequate. In addition, the study was prematurely stopped due to the limited number of inclusions. Indirectly, the randomised studies performed by VogELZANG et al. [63] and VAN MEERBEECK et al. [42] suggested that a polychemotherapy including cisplatin and an antifolate, pemetrexed or raltitrexed, could increase survival if we consider that cisplatin monotherapy is equivalent to a therapeutic abstention. Indeed, the median survival rates observed with the combinations of cisplatin-pemetrexed (12.1 months) or cisplatin-raltitrexed (11.4 months) were largely higher than those usually reported in the literature (7-9 months). The statistically significant difference in comparison with the cisplatin monotherapy arm (9.3 and 8.8 months) was an indirect argument suggesting a beneficial effect of chemotherapy. However, no published study has compared cisplatin monotherapy to palliative care only. 
Table 3 in the supplementary data summarises the data concerning chemotherapy in randomised (six trials) and nonrandomised studies. In first line, three randomised phase III trials are available [42, 62, 63]. They demonstrated the superiority of a combination of cisplatin and pemetrexed or raltitrexed over cisplatin monotherapy, both for response rates and survival, although cisplatin alone should not be considered as a standard treatment. It is important to note the role of folic acid and vitamin B12 supplementation to reduce the haematological toxicity of pemetrexed. Other cisplatin-based combinations produced interesting response rates, as observed in the meta-analysis of phase II studies [60, 61], approximately $25-30 \%$ for the following associations: cisplatin plus etoposide; cisplatin plus doxorubicin; cisplatin plus gemcitabine; cisplatin plus interferon; and oxaliplatin plus raltitrexed (or gemcitabine or vinorelbine) and methotrexate. The combinations cisplatinpemetrexed or cisplatin-raltitrexed could act as reference arms in further randomised trials. The inclusion of patients in good general condition in clinical trials remains ethically justified.

After failure of first-line chemotherapy, no randomised study demonstrated the impact of second-line treatment on survival or quality of life. Indirect data extracted from the follow-up of a first-line randomised trial [64] suggested that second-line chemotherapy after cisplatin-pemetrexed could increase survival in comparison with symptomatic treatment alone. These data need confirmation in a randomised study. The available data on this topic are rare (six phase II studies [65-70]) and do not allow proposal of a particular chemotherapy schedule. It is recommended to include patients in good general condition in clinical studies, with this approach being ethically acceptable.

First-line combination chemotherapy including cisplatin and pemetrexed or raltitrexed demonstrated greater activity than cisplatin alone in phase III trials (level 1), with higher response rates and improved survival. However, in the BTS study, there was no survival advantage of chemotherapy (vinorelbine alone or mitomycin $\mathrm{C}$, vinblastin and cisplatin combination) over best supportive care alone (level 2). Other studies, including potentially active combination, such as cisplatin plus gemcitabine or etoposide or doxorubicin, could be conducted (versus best supportive care or cisplatin/pemetrexed or raltitrexed) (expert opinion). The role of nonplatinum regimens remains to be elucidated (level 2). No randomised study has demonstrated the benefit of second-line chemotherapy on survival (except on survival without disease progression in a phase III study by JASSEM et al. [71]) or quality of life after failure of primary chemotherapy.

\section{Recommendations}

Every patient should receive at least best supportive care (grade 1A). When a decision is made to treat patients with chemotherapy, subjects in a good performance status (performance status $>60 \%$ on the Karnofsky scale or $<3$ on the Eastern Cooperative Oncology Group scale) should be treated with firstline combination chemotherapy consisting of platinum and pemetrexed or raltitrexed (grade 1B). Alternatively, patients could be included in first- and second-line clinical trials.

In the light of limited evidence of efficacy of chemotherapy, the decision to administer chemotherapy should be discussed with the patients and their relatives on a case-by-case basis, like all other treatment modalities without curative purposes (advice of experts).

When should chemotherapy be started, and for how long should chemotherapy be continued?

There is a shortage of available arguments in the literature on the most appropriate timing to administer chemotherapy. 1) An increase in overall survival in patients with adequate general condition was observed in two randomised phase III trials $[42,63] .2)$ Better theoretical efficacy of chemotherapy has been observed on small tumour volume [72-74]. 3) A small size randomised trial compared, in patients with controlled symptoms during at least 4 weeks, immediate chemotherapy versus delayed treatment at the time of symptoms progression. The duration before symptomatic progression (25 weeks versus 11 weeks) and survival (median 14 months versus 10 months; 1 yr $66 \%$ versus $36 \%$ ) were prolonged in the case of immediate chemotherapy without reaching formal statistical significance $(\mathrm{p}=0.1)[75]$.

There are no data allowing us to definitively answer the question of the optimal duration of chemotherapy. In the study by VoGELZANG et al. [63], 53\% of patients in the cisplatin-pemetrexed arm received six cycles (from one to 12 cycles, more than eight cycles in 5\%). In the study by van MEERBEECK et al. [42], the median number of cisplatin-raltitrexed cycles was five (from one to 10 cycles). We do not have data on the potential advantage of delivering more than six cycles in patients with stable disease. In comparison with nonsmall cell lung cancer, it is recommended to stop chemotherapy in case of progression, grade 3-4 toxicity or toxic cumulative doses, and to stop chemotherapy after six cycles in stable or responding patients. Experimental treatments, including biological therapies, must be discontinued according to the pre-specified experimental protocol. There are no data on the value of maintenance treatment with chemotherapy or immunomodulators.

\section{Recommendations}

Administration of chemotherapy should not be delayed and should be considered before the appearance of functional clinical signs (grade 1C).

Chemotherapy should be stopped in case of progressive disease, grade 3-4 toxicities or cumulative toxic doses (grade $1 \mathrm{~A})$, or following up to six cycles in patients who respond or who are stable (grade $2 \mathrm{C}$ ).

\section{What cytotoxic drugs are effective as second-line treatment?}

Several publications have specifically dealt with second-line chemotherapy [65-71, 76, 77]. Other articles are difficult to interpret because they assess patients receiving both first- and second-line treatment. These studies were not considered for this review. Chemotherapies consisting of doxorubicin, doxorubicin plus cyclophosphamide, oxaliplatin-raltitrexed or ZD 0473 (platinum analogue) appeared ineffective. Some interesting response rates were noted with pemetrexed alone [70], the combination of carboplatin and pemetrexed [70] and of cisplatin, irinotecan and mitomycine C [69]. Nevertheless, pemetrexed was compared in a phase III randomised trial versus best supportive care. It showed an improvement in response rate and time to progression but failed to show any survival benefit [77]. As vinorelbine has shown first-line 
activity it might be a reasonable choice for second-line treatment. A recent small study on 63 patients reported a $16 \%$ response rate and median survival of 9.6 months in this setting [77]. Thus, no drug has been validated in second-line chemotherapy and patients in a good performance status should be recommended to enter clinical trials instead.

\section{Recommendations}

Patients demonstrating prolonged symptomatic and objective response with first-line chemotherapy may be treated again with the same regimen in the event of recurrence (grade $2 \mathrm{C}$ ).

In other cases, inclusion of the patients in clinical trials is encouraged (grade 2C).

What is the role of biotherapies in the treatment of MPM?

Results of studies assessing the efficacy of drugs modulating the activity of the immune system or having a "specific" action on the tumour (targeted therapies) are summarised in table 3 of the supplementary data.

\section{Immunomodulators}

Interferons and interleukins (ILs) are the principal drugs being tested in the treatment of malignant mesothelioma. Dose, method of administration (intrapleural, sub-cutaneous, intramuscular and intravenous), type of drug and disease stage varied from one study to another, therefore, interpretation of the results must be performed cautiously. Monotherapy with interferons or IL-2 did not seem to effective and is not recommended outside of a clinical trial.

Interesting preliminary results were observed after administration of Mycobacterium vaccae in a limited number of patients. This treatment needs to be confirmed before its use can be recommended. Ranpirnase has not demonstrated its effectiveness.

\section{Targeted therapies}

Some biological targeted therapies are effective in lung, colon and breast cancers. Few studies are available in malignant mesothelioma. The principal drugs currently tested are as follows.

1) Thalidomide (anti-angiogenic drug): among 40 patients treated in a phase I/II trial, 11 presented with stable disease over $>6$ months, with median survival of 230 days. However, these results do not allow for the classification of thalidomide as an active drug [78]. 2) Bevacizumab (monoclonal antibody directed against vascular endothelial growth factor): a phase II randomised study compared cisplatin plus gemcitabine with or without bevacizumab. The addition of bevacizumab did not result in an improved response rate (25\% versus $22 \%$ ) or survival (mean survival time 15.6 months versus 14.7 months; $\mathrm{p}=0.91$ ) [79]. 3) Gefitinib: in a phase II study including 42 patients receiving gefitinib $500 \mathrm{mg}$ orally every day, only two objective responses were documented. The authors concluded the absence of activity of gefitinib in this indication [80]. 4) Imatinib: it demonstrated no activity in a published phase II trial [81] and in two studies presented at the American Society of Clinical Oncology meeting $[82,83]$. 5) Erlotinib: no objective response was observed in a phase II study among 33 patients with measurable disease [84].

\section{Recommendations}

Immunomodulating agents, targeted biotherapies and vaccines should not be used in the treatment of MPM outside clinical trials (grade 1C).

What assessment criteria should be used to determine the efficacy of chemotherapy in MPM?

The activity of a treatment can be assessed on clinical criteria (symptoms control and quality of life), imaging criteria (CT scan or PET) and survival criteria (time to progression and overall survival). The evaluation of response by thoracoscopy was never reported.

\section{Imaging evaluation criteria of tumour response}

Response evaluation criteria are vary from one study to another and are not always reported. The systematic practice of a referential CT scan after pleural symphysis and before beginning chemotherapy was not mandatory, distorting response evaluation. The timing for evaluation is also lacking most of the time.

Today, it can be considered that standard chest radiography is not a valuable method by which to assess response to chemotherapy (refer to Diagnosis section).

There are different methods for objective response assessment depending on the type of criteria, WHO (product of two perpendicular measures) or RECIST (one dimension measure). Neither of these methods is adapted to malignant mesothelioma in which development is essentially circumferential on the gross pleural surface [85]. It is currently proposed to use modified "RECIST criteria" (measure of the short diameter perpendicular to the chest wall court) to assess objective response in MPM [85-87].

\section{Tumour response evaluation according to PET criteria}

Differentiating tumour tissue from post-chemotherapy scar lesions is difficult with CT scanning. PET allows assessment of both tumour sizes and captation intensity. The combination of PET and CT scan, with both examinations performed on the patient in the same position, allows a better correlation of these two techniques. The contribution of this new imaging modality in response evaluation still needs to be validated. For clinical trials, in the absence of standardisation in response evaluation with PET in malignant mesothelioma, the use of the PET response criteria proposed by EORTC [88] can be considered (table 4 in supplementary data).

\section{Survival}

Overall survival is the only valuable criteria to assess the effectiveness of chemotherapy in therapeutic protocols.

\section{Quality of life}

It is recommended to take into account quality of life and symptom control to evaluate the clinical benefit (efficacy/ tolerance) in disease of poor prognosis and for which the survival impact of the treatment is not clearly demonstrated or marginal. No particular score to assess quality of life is specifically recommended except the modified version of the Lung Cancer Symptom Scale (LCSS), which is adapted to patients presenting with malignant mesothelioma [89]. 
Recommendations: For assessment and follow-up of MPM a chest CT scan is recommended. If a patient has had pleurodesis, a chest CT scan should be performed again before the start of chemotherapy in order to better evaluate the response to treatment (grade $1 \mathrm{~B}$ ).

The modified RECIST criteria are the preferred method of measuring response to treatment (grade 1B).

PET scan and biological markers are still under investigation for the evaluation of response to treatment in MPM.

\section{Combined modality approach}

The following criteria are considered for possible extra-pleural pneumonectomy (EPP) indications; 1) biopsy proven MPM of non-sarcomatoid cell type; 2 ) clinical and/or pathological stage T1-3, N0-1, M0 (it should be noted that some centres include patients with N2 disease in their study although N2 disease has a worse overall survival); 3) patient fit for pneumonectomy by virtue of sufficient respiratory reserve and lacking other comorbidity e.g. cardiovascular; 4) patient fit to receive neoadjuvant/adjuvant chemotherapy; 5) patient fit to receive adjuvant radical hemithorax irradiation; 6) EORTC and CALGB scores (table 6) may be calculated in patients to support EPP indication, but the value of the scores to define a "favourable prognosis" group should be validated in a prospective clinical trial.

What is the rationale behind the multi-modality approach?

Older literature indicates that surgery alone for MPM is not curative since no oncological resection margins can be obtained. The pleural lining, especially on the pericardium and mediastinum, cannot be resected with a 1-2-cm margin. Therefore, all surgical procedures are considered R1 resections [50]. This observation is, therefore, the rationale for combined therapy (level of evidence: strong/low-quality evidence).

The use of radiation therapy to the full hemithorax is limited by critical organs, such as contralateral lung, liver and heart most particularly, but also spinal cord and oesophagus. Therefore, it is difficult to administer a total dose of $>54$ Gy to such a large volume and, as such, sophisticated treatment techniques, oriented by findings from surgeons and pathologists, are needed [90,91] (level of evidence: strong/low-quality evidence).

\section{Which patient is suitable for this approach?}

Due to the extent of surgery and combination treatment, patients need to undergo a thorough work-up before embarking on any multi-modality treatment. Until 2004, most combined treatments focussed on surgery followed by radiation therapy since active chemotherapy regimens were not available. For potential patients the work-up should consist of at least the following. 1) Physical examination: shrinkage of the afflicted hemithorax is considered a sign of advanced disease. No signs of growth through the ribs or in the abdomen. 2) Pulmonary function tests: post-pneumonectomy values should be sufficient for normal daily life functioning. 3) Adequate cardiac reserve with the absence of elevated pulmonary pressure or rhythm disorders (level of evidence: weak/ moderate-quality evidence). 4) Radiological examinations to rule out spread of the disease beyond the rib cage through the diaphragm; contra-lateral extension and multiple node involvement (level of evidence: weak/moderate-quality evidence). 5) Histological examination: the best results have been obtained with MPM of the epithelial type (level of evidence: weak/high-quality evidence). 6) Sex: there are no solid data that there is a difference in response to treatment between the different sexes [92] (level of evidence: strong/low-quality evidence).

\section{What is the best combination?}

There is a body of literature that deals with the combination of surgical resection followed by radiation therapy. The procedures vary with regard to the extent of resection (removal of the complete diaphragm, pericardium, placement of patches, etc.). Recently, the bi-modality approach has been extended with pre- or per-operative chemotherapy: two studies have been performed using platinum with an anti-folate (pemetrexed). One has been presented as a poster [93] while the EORTC study 08031 is being analysed [94]. Some reports have been made on the use of pre- and post-operative chemotherapy combined with hyperthermia. This approach, however, is not tested in a multi-centre fashion [95-97]. All surgical combination therapies that included EPP can only be performed at the cost of additional morbidity $(\leqslant 70 \%)$ and a mortality rate in specialised centres that should be $<7 \%$ (level of evidence: strong/low-quality evidence).

Currently, national groups are considering the question as to whether there is any advantage at all of this tri-modality treatment. Recently, a Swiss study tested the effect of induction chemotherapy followed by EPP and limited radiation to highrisk sites in 61 patients. Of the 45 patients who had an EPP, the survival was 23 months compared with 19.8 months for the whole group [51] (level of evidence: strong/low-quality evidence). In conclusion, there are limited and weak data available on the best combination treatment.

\section{Recommendations}

Patients who are considered candidates for this multimodal approach should be included in a prospective randomised trial in specialised centres.

\section{Control of symptoms in MPM}

Mesothelioma has a high symptom burden: a study of 53 patients with mesothelioma receiving chemotherapy with cisplatin and gemcitabine revealed that their mean scores on the EORTC quality of life questionnaire exceeded reference scores in lung cancer in the following areas: fatigue, dyspnoea, pain, insomnia, cough and anorexia [98]. This section will discuss symptoms frequently experienced by patients with mesothelioma. A retrospective randomised notes review demonstrated the common symptoms in mesothelioma as summarised in table 5 of the supplementary data.

\section{Management of pain}

How is pain in MPM evaluated?

Pain in mesothelioma is frequently complex due to a combination of nociceptive, neuropathic and inflammatory factors [99].

Use of a visual analogue pain assessment tool improves cancer pain management (grade $1 C$ ). 
If the patient has cognitive impairment due to pain or advanced disease, pain may be assessed using a behavioural assessment tool such as the Doloplus scale (grade 1C).

What is the general principle of treatment of pain in MPM? Recommendations: Pain control in mesothelioma should follow the principles of cancer pain management (grade 1C).

However, due to the complex nature of pain in mesothelioma, adjunct analgesia may frequently be required in addition to opiates. In cases of refractory pain unresponsive to the usual measures, a specialist pain management or specialist palliative medicine opinion should be sought (grade 1C).

Occasionally neuroablative techniques may be required, depending on specialist advice, and with careful consideration of the risks and benefits (grade 2C).

Palliative radiotherapy may be proposed and effective in treating pain due to tumour nodules (grade 2C).

\section{Management of dyspnoea}

Is repeated pleural aspiration justified?

Recommendations: This should be avoidable if pleurodesis is performed early in the disease and before effusions have become loculated and/or the lung has become fixed and unable to expand fully (grade 1C).

Repeated aspiration or indwelling chest drain may occasionally be the most practical way to manage recurrent effusions in very frail patients (grade $2 \mathrm{C}$ ).

What is the place of pleurodesis?

Recommendation: Pleurodesis is useful in preventing recurrent effusions. Sterile talc is preferred to other agents (grade 1A).

\section{When should talc pleurodesis be performed?}

Recommendation: Pleurodesis is most effective when performed early in the disease process (grade 1C) but it should not be performed before sufficient tissue for diagnosis has been obtained (grade 1A).

Are other treatments of value in the management of dyspnoea? Recommendations: Low-dose oral morphine may be useful in reducing the sensation of dyspnoea and thus also reducing associated anxiety (grade 1A).

Oxygen may be helpful but should not be used unless there is evidence of reduced oxygen saturation (grade 1C).

\section{Can other measures be used to alleviate dyspnoea?}

A simple fan that creates a cool stream of air across the face may reduce the sensation of dyspnoea via the trigeminal nerve. Self-help breathlessness management techniques, designed to increase patients' sense of mastery over their breathlessness, have been shown to be effective in lung cancer but the work has not been conducted specifically in mesothelioma [100].

\section{Management of other physical symptoms}

This is a brief account of simple measures used to palliate common symptoms (advice of experts). Further information should be sought from expert texts on palliative medicine.

\section{Statements}

Cough may respond to cough suppressants such as codeine linctus or pholcodine. It is important to exclude or treat comorbidities such as chest infection or cardiac failure.

Anorexia, weight loss and fatigue constitute the anorexia/ cachexia syndrome common in many malignant conditions. Attention to high-energy, small-volume frequent meals, treatment of oral candida if present, and avoidance of dehydration and constipation may help.

Sweating may improve with avoidance of restrictive clothing, use of a fan and medication such as cimetidine.

Dysphagia may be due to oral candida or from external compression of the oesophagus due to tumour. Candida responds to treatment with oral fluconazole. Stenting of the oesophagus may be effective in reducing dysphagia due to external compression.

Ascites usually develops due to tumour extension through the diaphragm into the peritoneal cavity. Paracentesis may reduce discomfort due to large volume ascities but may need to be repeated.

Constipation results from inactivity, poor oral intake and as an inevitable consequence of opiates. Laxatives should be prescribed pro-actively and taken regularly. This sign may suggest a tumour extension through the diaphragm into the peritoneal cavity.

Vomiting may occur as a side-effect of chemotherapy and responds to anti-emetics. It may also be a side-effect of opiate analgesics and changing to an alternative may be successful.

\section{Management of psychological distress}

Patients with mesothelioma may exhibit anger, depression or stoicism and resigned acceptance. Reports from specific mesothelioma telephone help lines demonstrate that patients and their families request accurate information about the illness, treatment options, state benefits and medico-legal issues.

\section{Recommendations}

Support may be offered by specialist nurses, psychological or psychiatric services and asbestos support groups (grade 1C).

\section{STATEMENT OF INTEREST}

Statements of interest for A. Scherpereel and P. van Houtte can be found at www.erj.ersjournals.com/misc/statements.dtl

\section{ACKNOWLEDGEMENTS}

The affiliations for the Task Force members are as follows A. Scherpereel: Dept of Pulmonary and Thoracic Oncology, Hospital of the University (CHRU) of Lille II, Lille, France. P. Astoul: Dept of Pulmonary and Thoracic Oncology, Hôpital Sainte-Marguerite, Université de la Méditerranée, Marseille, France. P. Baas: Dept of Thoracic Oncology, The Netherlands Cancer Institute, Amsterdam, The Netherlands. T. Berghmans: Intensive Care and Dept of Thoracic Oncology, Institut Jules-Bordet, Brussels, Belgium. H. Clayson: Hospice of St. Mary of Furness, Ulverston, Cumbria. P. de Vuyst: Pulmonary Dept, Hôpital Erasme, Brussels, Belgium. H. Dienemann: Thoraxklinik am Universitätsklinikum, Heidelberg, Germany. F. Galateau-Salle: Dept of Pathology, CHRU de Caen, Caen, France. C. Hennequin: Dept of Cancérologie-radiothérapie, Hôpital Saint-Louis, 
Paris, France. G. Hillerdal: Lung Medicine, Karolinska Hospital, Stockholm, Sweden. C. Le Péchoux: Dept of Radiotherapy, Institut Gustave Roussy, Villejuif, France. L. Mutti: Oncologia Clinica, Vercelli, Italy. J-C. Pairon: INSERM, Unit 955, Créteil, France. R. Stahel: Policlinic of Oncology, University Hospital of Zurich, Zurich, Switzerland. P. van Houtte: Dept de Radio-oncologie, Institut Jules-Bordet, Brussels, Belgium. J. van Meerbeeck: Dept of Respiratory Medicine and Thoracic Oncology, University Hospital, Gent, Belgium. D. Waller: Dept of Thoracic Surgery, Glenfield Hospital, Leicester, UK. W. Weder: University Hospital, Division of Thoracic Surgery, Zurich, Switzerland.

\section{REFERENCES}

1 Robinson BW, Musk AW, Lake RA. Malignant mesothelioma. Lancet 2005; 366: 397-408.

2 Hodgson JT, McElvenny DM, Darnton AJ, et al. The expected burden of mesothelioma mortality in Great Britain from 2002 to 2050. Br J Cancer 2005; 92: 587-593.

3 Pass HI, Vogelzang N, Hahn S, et al. Malignant pleural mesothelioma. Curr Probl Cancer 2004; 28: 93-174.

4 Scherpereel A. Guidelines of the French Speaking Society for Chest Medicine for management of malignant pleural mesothelioma. Respir Med 2007; 101: 1265-1276.

5 Scherpereel A. [The experts' conference of the Societe de Pneumologie de Langue Francaise (SPLF) on malignant pleural mesothelioma (MPM): useful and necessary recommendations] Rev Mal Respir 2006; 23: 11S5-11S6.

6 Stahel RA, Weder W, Felip E. Malignant pleural mesothelioma: ESMO clinical recommendations for diagnosis, treatment and follow-up. Ann Oncol 2008; 19: Suppl. 2, ii43-ii44.

7 British Thoracic Society Standards of Care Committee, BTS statement on malignant mesothelioma in the UK, 2007. Thorax 2007; 62: Suppl. 2, ii1-ii19.

8 Guyatt G, Gutterman D, Baumann MH, et al. Grading strength of recommendations and quality of evidence in clinical guidelines: report from an American College Of Chest Physicians task force. Chest 2006; 129: 174-181.

9 Wagner JC, Sleggs CA, Marchand P. Diffuse pleural mesothelioma and asbestos exposure in the North Western Cape Province. Br J Ind Med 1960; 17: 260-271.

10 Goldberg M, Imbernon E, Rolland P, et al. The French National Mesothelioma Surveillance Program. Occup Environ Med 2006; 63: 390-395.

11 Bourdes V, Boffetta P, Pisani P. Environmental exposure to asbestos and risk of pleural mesothelioma: review and metaanalysis. Eur J Epidemiol 2000; 16: 411-417.

12 Maule MM, Magnani C, Dalmasso P, et al. Modeling mesothelioma risk associated with environmental asbestos exposure. Environ Health Perspect 2007; 115: 1066-1071.

13 Iwatsubo Y, Pairon JC, Boutin C, et al. Pleural mesothelioma: dose-response relation at low levels of asbestos exposure in a French population-based case-control study. Am J Epidemiol 1998; 148: 133-142.

14 Lanphear BP, Buncher CR. Latent period for malignant mesothelioma of occupational origin. J Occup Med 1992; 34: 718-721.

15 Reid A, de Klerk N, Ambrosini G, et al. The additional risk of malignant mesothelioma in former workers and residents of Wittenoom with benign pleural disease or asbestosis. Occup Environ Med 2005; 62: 665-659.

16 Roushdy-Hammady I, Siegel J, Emri S, et al. Genetic-susceptibility factor and malignant mesothelioma in the Cappadocian region of Turkey. Lancet 2001; 357: 444-445.

17 Dogan AU, Baris YI, Dogan M, et al. Genetic predisposition to fiber carcinogenesis causes a mesothelioma epidemic in Turkey. Cancer Res 2006; 66: 5063-5068.
18 Lin RT, Takahashi K, Karjalainen A, et al. Ecological association between asbestos-related diseases and historical asbestos consumption: an international analysis. Lancet 2007; 369: 844-849.

19 Robinson BW, Lake RA. Advances in malignant mesothelioma. N Engl J Med 2005; 353: 1591-1603.

20 Segura O, Burdorf A, Looman C. Update of predictions of mortality from pleural mesothelioma in the Netherlands. Occup Environ Med 2003; 60: 50-55.

21 Montanaro F, Bray F, Gennaro V, et al. Pleural mesothelioma incidence in Europe: evidence of some deceleration in the increasing trends. Cancer Causes Control 2003; 14: 791-803.

22 Pelucchi C, Malvezzi M, La Vecchia C, et al. The Mesothelioma epidemic in Western Europe: an update. Br J Cancer 2004; 90: 1022-1024.

23 De Vuyst P, Karjalainen A, Dumortier P, et al. Guidelines for mineral fibre analyses in biological samples: report of the ERS Working Group. European Respiratory Society. Eur Respir J 1998; 11: 1416-1426.

24 Fasola G, Belvedere O, Aita M, et al. Low-dose computed tomography screening for lung cancer and pleural mesothelioma in an asbestos-exposed population: baseline results of a prospective, nonrandomized feasibility trial - an Alpe-adria Thoracic Oncology Multidisciplinary Group Study (ATOM 002). Oncologist 2007; 12: 1215-1224.

25 Scherpereel A, Lee YC. Biomarkers for mesothelioma. Curr Opin Pulm Med 2007; 13: 339-443.

26 Park EK, Sandrini A, Yates DH, et al. Soluble mesothelin-related protein in an asbestos-exposed population: the dust diseases board cohort study. Am J Respir Crit Care Med 2008; 178: 832-837.

27 van Meerbeeck JP, Hillerdal G. Screening for mesothelioma: more harm than good? Am J Respir Crit Care Med 2008; 178: 781-782.

28 Eibel R, Tuengerthal S, Schoenberg SO. The role of new imaging techniques in diagnosis and staging of malignant pleural mesothelioma. Curr Opin Oncol 2003; 15: 131-138.

29 Wang ZJ, Reddy GP, Gotway MB, et al. Malignant pleural mesothelioma: evaluation with CT, MR imaging, and PET. Radiographics 2004; 24: 105-119.

30 Francis RJ, Byrne MJ, van der Schaaf AA, et al. Early prediction of response to chemotherapy and survival in malignant pleural mesothelioma using a novel semiautomated 3-dimensional volume-based analysis of serial 18F-FDG PET scans. J Nucl Med 2007; 48: 1449-1458.

31 Flores RM. The role of PET in the surgical management of malignant pleural mesothelioma. Lung Cancer 2005; 49: Suppl. 1, S27-S32.

32 Travis WD, Brambilla E, Muller-Hermelink HK, et al, eds. World Health Organization Classification of Tumours. Pathology and Genetics. Tumours of the Lung, Pleura, Thymus and Heart. Lyon, IARC, 2004.

33 Wittekind Ch, Greene FL, Hutter RVP, et al., eds. UICC, International Union Against Cancer. TNM Atlas. Illustrated Guide to the TMN Classification of Malignant Tumours. 5th Edn, Berlin, Springer, 2004; pp. 169-176.

34 van Meerbeeck JP, Boyer M. Consensus report: pretreatment minimal staging and treatment of potentially resectable malignant pleural mesothelioma. Lung Cancer 2005; 49: Suppl. 1, S123-S127.

35 Steele JP. Prognostic factors for mesothelioma. Hematol Oncol Clin North Am 2005; 19, 1041-1052: vi.

36 Spirtas R, Connelly RR, Tucker MA. Survival patterns for malignant mesothelioma: the SEER experience. Int $J$ Cancer 1988; 41: 525-530.

37 Robinson BW, Creaney J, Lake R, et al. Mesothelin-family proteins and diagnosis of mesothelioma. Lancet 2003; 362: 1612-1616.

38 Pass HI, Lott D, Lonardo F, et al. Asbestos exposure, pleural mesothelioma, and serum osteopontin levels. N Engl J Med 2005; 353: 1564-1573. 
39 Grigoriu BD, Scherpereel A, Devos P, et al. Utility of osteopontin and serum mesothelin in malignant pleural mesothelioma diagnosis and prognosis assessment. Clin Cancer Res 2007; 13: 2928-2935.

40 Herndon JE, Green MR, Chahinian AP, et al. Factors predictive of survival among 337 patients with mesothelioma treated between 1984 and 1994 by the Cancer and Leukemia Group B. Chest 1998; 113: 723-731.

41 Curran D, Sahmoud T, Therasse $\mathrm{P}$, et al. Prognostic factors in patients with pleural mesothelioma: the European Organization for Research and Treatment of Cancer experience. J Clin Oncol 1998; 16: 145-152.

42 van Meerbeeck JP, Gaafar R, Manegold C, et al. Randomized phase III study of cisplatin with or without raltitrexed in patients with malignant pleural mesothelioma: an intergroup study of the European Organisation for Research and Treatment of Cancer Lung Cancer Group and the National Cancer Institute of Canada. J Clin Oncol 2005; 23: 6881-6889.

43 Bottomley A, Coens C, Efficace F, et al. Symptoms and patientreported well-being: do they predict survival in malignant pleural mesothelioma? A prognostic factor analysis of EORTCNCIC 08983: randomized phase III study of cisplatin with or without raltitrexed in patients with malignant pleural mesothelioma. J Clin Oncol 2007; 25: 5770-5776.

44 Waller DA, Morritt GN, Forty J. Video-assisted thoracoscopic pleurectomy in the management of malignant pleural effusion. Chest 1995; 107: 1454-1456.

45 Soysal O, Karaoglanoglu N, Demiracan S, et al. Pleurectomy/ decortication for palliation in malignant pleural mesothelioma: results of surgery. Eur J Cardiothorac Surg 1997; 11: 210-213.

46 Halstead JC, Lim E, Venkateswaran RM, et al. Improved survival with VATS pleurectomy-decortication in advanced malignant mesothelioma. Eur J Surg Oncol 2005; 31: 314-320.

47 Nakas A, Martin Ucar AE, Edwards JG, et al. The role of video assisted thoracoscopic pleurectomy/decortication in the therapeutic management of malignant pleural mesothelioma. Eur J Cardiothorac Surg 2008; 33: 83-88.

48 Martin-Ucar AE, Edwards JG, Rengajaran A, et al. Palliative surgical debulking in malignant mesothelioma. Predictors of survival and symptom control. Eur J Cardiothorac Surg 2001; 20: 1117-1121.

49 Sugarbaker DJ, Jaklitsch MT, Bueno R, et al. Prevention, early detection, and management of complications after 328 consecutive extrapleural pneumonectomies. J Thorac Cardiovasc Surg 2004; 128: 138-146.

50 Rusch VW, Rosenzweig K, Venkatraman E, et al. A phase II trial of surgical resection and adjuvant high-dose hemithoracic radiation for malignant pleural mesothelioma. I Thorac Cardiovasc Surg 2001; 122: 788-795.

51 Weder W, Stahel RA, Bernhard J, et al. Multicenter trial of neoadjuvant chemotherapy followed by extrapleural pneumonectomy in malignant pleural mesothelioma. Ann Oncol 2007; 18: 1196-1202.

52 Boutin C, Rey F, Viallat JR. Prevention of malignant seeding after invasive diagnostic procedures in patients with pleural mesothelioma. A randomized trial of local radiotherapy. Chest 1995; 108: 754-758.

53 O'Rourke N, Garcia JC, Paul J, et al. A randomised controlled trial of intervention site radiotherapy in malignant pleural mesothelioma. Radiother Oncol 2007; 84: 18-22.

54 Muirhead R, O'Rourke N. Drain site radiotherapy in malignant pleural mesothelioma: a wasted resource. Eur Respir J 2007; 30: 1021.

55 Chapman E, Berenstein E, Diéguez M, et al. Radiotherapy for malignant pleural mesothelioma. Cochrane Database Syst Rev 2006; 3: CD003880.
56 Baldini EH. External beam radiation therapy for the treatment of pleural mesothelioma. Thorac Surg Clin 2004; 14: 543-548.

57 Allen AM, Czerminska M, Janne PA, et al. Fatal pneumonitis associated with intensity-modulated radiation therapy for mesothelioma. Int J Radiat Oncol Biol Phys 2006; 65: 640-645.

58 Berghmans T, Brechot JM, Robinet G, et al. [Chemotherapy of malignant pleural mesothelioma.] Rev Mal Respir 2006; 23: 11S57-11S70.

59 Ellis P, Davies AM, Evans WK, et al. The Use of Chemotherapy in Patients with Advanced Malignant Pleural Mesothelioma. A Quality Initiative of the Program in Evidence-based Care (PEBC), Cancer Care Ontario (CCO). October 2005. www. cancercare.on.ca/pdf/pebc7-14-1f.pdf

60 Berghmans T, Paesmans M, Lalami Y, et al. Activity of chemotherapy and immunotherapy on malignant mesothelioma: a systematic review of the literature with meta-analysis. Lung Cancer 2002; 38: 111-121.

61 Berghmans T LJ, Mascaux C, Meert AP, et al. Activity of chemotherapy and immunotherapy on malignant mesothelioma: a systematic review of the literature with meta-analysis, 2003 245-256.

62 Muers MF, Stephens RJ, Fisher P, et al. Active symptom control with or without chemotherapy in the treatment of patients with malignant pleural mesothelioma (MS01): a multicentre randomised trial. Lancet 2008; 371: 1685-1694.

63 Vogelzang NJ, Rusthoven JJ, Symanowski J, et al. Phase III study of pemetrexed in combination with cisplatin versus cisplatin alone in patients with malignant pleural mesothelioma. J Clin Oncol 2003; 21: 2636-2644.

64 Manegold C, Symanowski J, Gatzemeier U, et al. Second-line (post-study) chemotherapy received by patients treated in the phase III trial of pemetrexed plus cisplatin versus cisplatin alone in malignant pleural mesothelioma. Ann Oncol 2005; 16: 923-927.

65 Giaccone G, O'Brien ME, Byrne MJ, et al. Phase II trial of ZD0473 as second-line therapy in mesothelioma. Eur J Cancer 2002; 38: Suppl. 8, S19-S24.

66 Harvey VJ, Slevin ML, Ponder BA, et al. Chemotherapy of diffuse malignant mesothelioma. Phase II trials of single-agent 5fluorouracil and adriamycin. Cancer 1984; 54: 961-964.

67 Porta C, Zimatore M, Bonomi L, et al. Raltitrexed-oxaliplatin combination chemotherapy is inactive as second-line treatment for malignant pleural mesothelioma patients. Lung Cancer 2005 48: 429-434

68 Sorensen PG, Bach F, Bork E, et al. Randomized trial of doxorubicin versus cyclophosphamide in diffuse malignant pleural mesothelioma. Cancer Treat Rep 1985; 69: 1431-1432.

69 Fennell DA, Steele JP, Shamash J, et al. Efficacy and safety of first- or second-line irinotecan, cisplatin, and mitomycin in mesothelioma. Cancer 2007; 109: 93-99.

70 Sorensen JB, Sundstrom S, Perell K, et al. Pemetrexed as second-line treatment in malignant pleural mesothelioma after platinumbased first-line treatment. J Thorac Oncol 2007; 2: 147-152.

71 Jassem J, Ramlau R, Santoro A, et al. Phase III trial of pemetrexed plus best supportive care compared with best supportive care in previously treated patients with advanced malignant pleural mesothelioma. J Clin Oncol 2008; 26: 1698-1704.

72 DeVita VT Jr. The James Ewing lecture. The relationship between tumor mass and resistance to chemotherapy. Implications for surgical adjuvant treatment of cancer. Cancer 1983; 51: 1209-1220.

73 Skipper HE, Schabel FM Jr, Wilcox WS. Experimental evaluation of potential anticancer agents. Xiii. on the criteria and kinetics associated with "curability" of experimental leukemia. Cancer Chemother Rep 1964; 35: 1-111.

74 Skipper HE. Laboratory models: some historical perspective. Cancer Treat Rep 1986; 70: 3-7.

75 O'Brien ME, Watkins D, Ryan C, et al. A randomised trial in malignant mesothelioma (M) of early (E) versus delayed (D) 
chemotherapy in symptomatically stable patients: the MED trial. Ann Oncol 2006; 17: 270-275.

76 Ramalingam SS, Belani CP, Ruel C, et al. Phase II study of belinostat (PXD101), a histone deacetylase inhibitor, for second line therapy of advanced malignant pleural mesothelioma. J Thorac Oncol 2009; 4: 97-101.

77 Stebbing J, Powles T, McPherson K, et al. The efficacy and safety of weekly vinorelbine in relapsed malignant pleural mesothelioma. Lung Cancer 2009; 63: 94-97.

78 Baas $\mathrm{P}$, Boogerd W, Dalesio O, et al. Thalidomide in patients with malignant pleural mesothelioma. Lung Cancer 2005; 48: 291-296.

79 Karrison TKH, Gandara DR, Lu C, et al. Final analysis of a multicenter, double-blind, placebo-controlled, randomized phase II trial of gemcitabine/cisplatin (GC) plus bevacizumab (B) or placebo $(\mathrm{P})$ in patients (pts) with malignant mesothelioma (MM). J Clin Oncol 2007; 25: Suppl. 20, 18 S.

80 Govindan R, Kratzke RA, Herndon JE 2nd, et al. Gefitinib in patients with malignant mesothelioma: a phase II study by the Cancer and Leukemia Group B. Clin Cancer Res 2005; 11: 2300-2304.

81 Mathy A, Baas P, Dalesio O, et al. Limited efficacy of imatinib mesylate in malignant mesothelioma: a phase II trial. Lung Cancer 2005; 50: 83-86.

82 Millward MPF, Byrne M, Powell A, et al. Phase II trial of imatinib mesylate in patients with advanced pleural mesothelioma. Proc Am Soc Clin Oncol 2003; 22: 228.

83 Villano JL, Hussain AN, Stadler WM, et al. A phase II trial of imatinib mesylate in patients with malignant mesothelioma. Proc Am Soc Clin Oncol 2004; 22: Suppl. 15, 14s.

84 Garland LL, Rankin C, Gandara DR, et al. Phase II study of erlotinib in patients with malignant pleural mesothelioma: a Southwest Oncology Group Study. J Clin Oncol 2007; 25: 2406-2413.

85 van Klaveren RJ, Aerts JG, de Bruin H, et al. Inadequacy of the RECIST criteria for response evaluation in patients with malignant pleural mesothelioma. Lung Cancer 2004; 43: 63-69.

86 Byrne MJ, Nowak AK. Modified RECIST criteria for assessment of response in malignant pleural mesothelioma. Ann Oncol 2004; 15: 257-260.

87 Nowak AK. CT, RECIST, and malignant pleural mesothelioma. Lung Cancer 2005; 49: Suppl. 1, S37-S40.

88 Young H, Baum R, Cremerius U, et al. Measurement of clinical and subclinical tumour response using [18F]-fluorodeoxyglucose and positron emission tomography: review and 1999 EORTC recommendations. European Organization for Research and Treatment of Cancer (EORTC) PET Study Group. Eur J Cancer 1999; 35: 1773-1782.

89 Hollen PJ, Gralla RJ, Liepa AM, et al. Measuring quality of life in patients with pleural mesothelioma using a modified version of the Lung Cancer Symptom Scale (LCSS): psychometric properties of the LCSS-Meso. Support Care Cancer 2006; 14: 11-21.

90 Senan S. Indications and limitations of radiotherapy in malignant pleural mesothelioma. Curr Opin Oncol 2003; 15: 144-147.

91 Maasilta P. Deterioration in lung function following hemithorax irradiation for pleural mesothelioma. Int J Radiat Oncol Biol Phys 1991; 20: 433-438.

92 Steele JP, Klabatsa A, Fennell DA, et al. Prognostic factors in mesothelioma. Lung Cancer 2005; 49: Suppl. 1, S49-S52.

93 Krug LMK, Pass H, Rusch VW, et al. A multicenter U.S. trial of neoadjuvant pemetrexed plus cisplatin (PC) followed by extrapleural pneumonectomy (EPP) and hemithoracic radiation (RT) for stage I-III malignant pleural mesothelioma (MPM). Proc Am Soc Clin Oncol 2007; 25: Suppl. 20, 18 S.

94 Van Schil P, Hasan B, Maat A, et al. EORTC 08031. The 9th international conference of the International Mesothelioma Interest Group. Amsterdam, The Netherlands, 2008: p. 174.

95 Monneuse $\mathrm{O}$, Beaujard AC, Guibert B, et al. Long-term results of intrathoracic chemohyperthermia (ITCH) for the treatment of pleural malignancies. Br J Cancer 2003; 88: 1839-1843.

96 van Ruth S, Baas P, Haas RL, et al. Cytoreductive surgery combined with intraoperative hyperthermic intrathoracic chemotherapy for stage I malignant pleural mesothelioma. Ann Surg Oncol 2003; 10: 176-182.

97 Xia H, Karasawa K, Hanyu N, et al. Hyperthermia combined with intra-thoracic chemotherapy and radiotherapy for malignant pleural mesothelioma. Int J Hyperthermia 2006; 22: 613-621.

98 Nowak AK, Stockler MR, Byrne MJ. Assessing quality of life during chemotherapy for pleural mesothelioma: feasibility, validity, and results of using the European Organization for Research and Treatment of Cancer Core Quality of Life Questionnaire and Lung Cancer Module. J Clin Oncol 2004; 22: 3172-3180.

99 Ahmedzai S, Clayson H. Palliative care for mesothelioma. In: O'Byrne K, Rusch V, eds. Malignant Pleural Mesothelioma. Oxford, Oxford University Press, 2006: pp. 412-418.

100 Corner J, Plant H, A'Hern R, et al. Non-pharmacological intervention for breathlessness in lung cancer. Palliat Med 1996; 10: 299-305. 